\title{
Imaging Solid-Electrolyte-Interphase Dynamics Using In-operando Reflection Interference Microscopy
}

\section{Guangxia Feng}

University of Houston

Hao Jia

Pacific Northwest National Laboratory

Yaping Shi

University of Houston

\section{Xu Yang}

University of Houston

Ye Zhang

University of Houston

Chaojie Yang

University of Houston

\section{Kang Xu}

U.S. Army Research Laboratory

Yan Yao

University of Houston https://orcid.org/0000-0002-8785-5030

Wu Xu

Pacific Northwest National Laboratory https://orcid.org/0000-0002-2685-8684

Xiaonan Shan ( $\nabla$ xshan@central.uh.edu )

University of Houston

\section{Article}

Keywords:

Posted Date: March 7th, 2022

DOI: https://doi.org/10.21203/rs.3.rs-1390037/v1

License: (1) (1) This work is licensed under a Creative Commons Attribution 4.0 International License.

Read Full License 


\title{
Imaging Solid-Electrolyte-Interphase Dynamics Using In-operando Reflection Interference Microscopy
}

Guangxia Feng ${ }^{1}$, Hao $\mathrm{Jia}^{2}$, Yaping Shi ${ }^{1}$, Xu Yang ${ }^{1}$, Ye Zhang ${ }^{3}$, Chaojie Yang ${ }^{1}$, Kang Xu ${ }^{4 *}$, Yan Yao ${ }^{1,3,5 *}$, $\mathrm{Wu} \mathrm{Xu}{ }^{2 *}$, Xiaonan $\mathrm{Shan}^{1 *}$

${ }^{1}$ Electrical and Computer Engineering Department, University of Houston, Houston, TX 77204, USA

${ }^{2}$ Energy and Environment Directorate, Pacific Northwest National Laboratory, Richland, WA 99354, USA

${ }^{3}$ Materials Science and Engineering Program, University of Houston, Houston, TX 77204, USA

${ }^{4}$ Battery Science Branch, Energy Science Division, Sensor and Electron Devices Directorate, Army Research Laboratory, Adelphi, MD, USA

${ }^{5}$ Texas Center for Superconductivity at the University of Houston, University of Houston, Houston, TX, 77204, USA

\section{Corresponding Authors}

*E-mails: conrad.k.xu.civ@army.mil ; yyao4@uh.edu ; wu.xu@pnnl.gov ; xshan@central.uh.edu;

\begin{abstract}
The quality of solid-electrolyte-interphase (SEI) dictates the performances of most battery chemistries, especially lithium ( $\mathrm{Li}$ )-metal, but its formation processes as well as evolution during battery operation remain little understood due to the lack of reliable in-operando characterization tools of sufficient spatial and temporal resolutions. Herein, we report an in-operando reflection interference microscope (RIM) that enables the real-time imaging of SEI during formation and evolution in state-of-the-art electrolyte based on $\mathrm{LiPF}_{6}$ dissolved in organic carbonates. By mapping the minimal and localized optical signals generated from interphasial events, RIM reveals with extremely high sensitivity that the stratified structure of SEI formed during four distinct steps, including the emergence of a permanent inner inorganic layer enriched in $\mathrm{LiF}$, the transient assembly of an interfacial structure of an electrified double layer, and the consequent emergence of a temporary outer organic-rich layer, whose presence is reversible with electrochemical cycling. Comparing the morphologies of SEIs, we identify an inverse correlation between the thicknesses of two interphasial sub-components: the thicker the LiF-rich inner layer, the thinner the organic-rich outer layer, implying that the permanent inorganic-rich inner layer dictates the organic-rich outer layer formation and $\mathrm{Li}$ nucleation. We also find that trace presence of water $(50 \mathrm{ppm})$ in the electrolyte induces a much thicker and higher quality LiF-rich layer and a much thinner organic-rich layer in SEI, which leads to less electrolyte consumption, and more uniform $\mathrm{Li}$ nucleation on the electrode surface. The real-time visualization of SEI dynamics achieved for the first time in this work provides a guideline for the rational design of interphases, a battery component that has been the least understood and most challenging barrier to developing electrolytes for future batteries.
\end{abstract}




\section{Introduction}

Lithium (Li)-ion batteries (LIBs) have transformed modern electronic devices and are universally used for electric vehicles and grid energy storage industry ${ }^{1,2,3,4,5}$. However, the demand for higher specific energy density from batteries has been persistently pressing for new battery chemistries to be developed ${ }^{6,7,2,8,9,10 \text {, }}$ 11, 12. Li metal $\left(\mathrm{Li}^{0}\right)$ anode promises to be the ultimate anode material due to the combination of its ultrahigh theoretical specific capacity and the lowest electrochemical redox potential, while an "anode-free" configuration that generates in-situ $\mathrm{Li}^{0}$ anode makes the goal of $500 \mathrm{Wh} / \mathrm{Kg}$ possible ${ }^{13}, 14,15,16$. The interphase in anode-free cells differs from those formed in either conventional LIBs or Li-metal batteries (LMBs), with mixed characteristics of both stepwise/discriminating nature of the former, and instantaneous/indiscriminating nature of the latter ${ }^{17}$. Hence it presents as the most interesting and meaningful case for a fundamental understanding of interphase. In such cells, during the initial charging process, the potential of the bare copper $(\mathrm{Cu})$ foil is brought progressively below the lowest unoccupied molecular orbital (LUMO) energy of the electrolyte, thus triggering an irreversible reaction, forming the primitive solid-electrolyte interphase (SEI). This proto-SEI experiences further evolutions as the $\mathrm{Cu}$ foil is polarized more cathodically, while the emergence of $\mathrm{Li}^{0}$ at lower potential induces instantaneous reactions with bulk electrolyte, forming the second round of interphasial chemicals. Such an interphase on $\mathrm{Cu}$ and $\mathrm{Li}^{0}$ surfaces stabilizes electrolyte against the reductive decomposition by blocking electron tunneling while still allowing $\mathrm{Li}^{+}$to migrate, so that the battery operates reversibly without sustained electrolyte decomposition $^{18,19,20}$. Therefore, SEI holds the key to the next generation high energy batteries.

To understand the physical and chemical properties of the SEI formed in LIBs and LMBs, various advanced analytical techniques have been developed and explored. Ex-situ studies including scanning electron microscopy $(\mathrm{SEM})^{21}$, transmission electron microscopy (TEM) ${ }^{22,}{ }^{23}$, Fourier-transform infrared (FTIR) spectroscopy ${ }^{24,25,26}$, Raman spectroscopy ${ }^{27,28,29}$, X-ray photoelectron spectroscopy (XPS) ${ }^{30,31,32}$ reveal important local information about structural and chemical natures of SEIs. However, high-energy electron and X-ray beam inevitably induce damage to the fragile SEI, creating artifacts. In-situ secondary ion mass spectrometry (SIMS) ${ }^{33}$ provides precise identification of SEI chemical components, however, structural and morphological information is missing. Recently, inspired by biological imaging techniques, ex-situ cryogenic electron microscopy (cryo-EM) has been successfully applied to preserve the native state of SEI during the high-resolution electron imaging at cryogenic conditions ${ }^{34,35}$. This technique made the atomic-resolution imaging of SEI layers and $\mathrm{Li}$ dendrites possible, although it still only reveals static and cross-sectional information while completely missing the dynamic evolution of SEI during charge/discharge cycles. In-situ atomic force microscopy (AFM) provides vivid morphological changes of the $\mathrm{SEI}^{36,37,38}$, however, its relatively slow scan speed limits its capability of mapping the dynamic processes, while chemical information is also missing. Finally, electrochemical quartz crystal microbalance (EQCM) accurately measures mass change caused by the SEI formation ${ }^{39}$, but without local information regarding the distribution of the chemical components, which might be critical to evaluate the SEI quality. Most importantly, the peculiar "hybrid" complexity of interphases formed in anode-free LMBs has never been recognized, let alone investigated or understood.

In this work, we attempt to fill in the gap left by the current techniques. By applying an in-operando reflection interference microscope (RIM) with high sensitivity as well as a high temporal and spatial resolution on a $\mathrm{Li}^{0}$-halfcell that simulates the anode of "anode-free" $\mathrm{LMB}$, we directly image and map the formation dynamics of SEI on $\mathrm{Cu}$ surface and the subsequent transformation at the emergence of $\mathrm{Li}^{0}$, and its evolutions over cycles. The interference created by the top and bottom surfaces of various SEI strata provides high sensitivity to detect minimal signals generated from any interfacial events. The electrolyte consisting of $1 \mathrm{M} \mathrm{LiPF}_{6}$ in propylene carbonate (PC) was adopted as the benchmark because it has been well-studied by various techniques, whose chemical pathways have been well established ${ }^{40,41}$. Using the RIM, we successfully observed the entire evolution process of SEI layer in real time. We find that the SEI layer's formation and stripping experiences a multi-step process that involves the formation of a proto-SEI based on LiF-containing inorganic layer, the assembly of an electrical double layer (EDL) interface, and 
the reversible emergence and disappearance of a transient organic-rich SEI layer. The formation of this LiF-rich inner layer dominates the process in the first cycle, and it evolves slowly during the consecutive cycles into a permanent interphase layer. However, the organic-rich outer layer emerges after the formation of LiF-rich proto-SEI, and disappears in each cycle depending on the electrode potential. We also observed the continuous charge accumulation/depletion at EDL even after the formation of the permanent LiF-rich SEI layer, which will not block the migration of $\mathrm{Li}^{+}$. Since RIM provides a unique capability that allows us to differentiate the signals generated from the LiF-rich inner layer and the organic-rich outer layer formation, we are able to translate the optical signals into localized morphology maps of LiF-rich inner layer and organic-rich outer layer, separately, and hence quantitatively revealed the strong inverse correlation between the thicknesses of the LiF-rich inner layer and the organic-rich outer layer, i.e., the thicker the LiFrich inner layer, the thinner the organic-rich outer layer. This correlation is further confirmed in the trace presence of water, which is deliberately added to promote HF-generation and eventually leads to a much thicker and more uniform LiF-rich inner layer along with minimized electrolyte consumption and more uniform Li nucleation on the electrode surface. The knowledge of the dynamic evolution of SEI upon cell cycling and electrolyte additives provides useful guidelines in tailor-design interphases for batteries of better performances.

\section{Imaging local surface reactions with RIM}

In the initial charge, the potential of $\mathrm{Cu}$ is progressively polarized cathodically, and the electrolyte component (solvent, salt and additive) with the highest LUMO will be preferentially reduced generating the primitive chemical ingredients for the proto-SEI, which experiences further electrochemical reduction as $\mathrm{Cu}$ continues the cathodic polarization, till $\mathrm{Li}^{0}$ is deposited and the eventual permanent interphase is formed. An in-situ and in-operando imaging method RIM is hence applied to map the local emergence and distribution of SEI components generated in the above process, and more importantly, to probe the dynamics of the deposition and stripping of the extremely reactive $\mathrm{Li}^{0}$. Fig. 1a schematically displays the working principle of RIM. The reflected light at the electrode/electrolyte junction, which would later become electrode/SEI and SEI/electrolyte junctions after SEI is formed, respectively (Fig. 1a right), will interfere with each other, which, after proper translation, carries information about the phase delay created by the changes in SEI layer (Figs. 1b,c). If a layer deposited on the electrode surface has a refractive index smaller than the surrounding electrolyte, the reflected optical intensity will increase, otherwise, it will decrease (Supplementary Section 4).

To image the SEI formation on the electrode surface, a three-electrode system is used (Fig. 1a), where the $\mathrm{Cu}$ foil serves as the working electrode, and two Li wires as the reference and the counter electrodes, respectively. Such a halfcell simulates the anode side of the "anode-free" LMB. The cell was first cycled within the voltage range of $2.3 \mathrm{~V}$ to $0.1 \mathrm{~V}$ vs. $\mathrm{Li} / \mathrm{Li}^{+}$, in which the SEI is formed in a stepwise and discriminating manner, similar to what happens in the initial cycles of LIBs.

\section{Formation of LiF-rich proto-SEI}

The optical reflectance of the $\mathrm{Cu}$ surface changes significantly in the first 3 cycles of cyclic voltametric (CV) scans (Fig. 1c), which is strongly correlated with the applied potential and the electrochemical current response (red and black curves in Fig. 1d, respectively). At the beginning of the first cycle $(0 \sim 5 \mathrm{~s})$, a reflectance decrease (marked by the black arrow in Fig. 1c) and a corresponding current spike are observed (marked by the black arrow in Fig. 1d), which is attributed to the reduction of $\mathrm{Cu}$ oxide $(\mathrm{CuO})$ on the $\mathrm{Cu}$ electrode surface that is prevalent product by ambient $\mathrm{air}^{42}$. After the $\mathrm{CuO}$ reduction, a current appears between $2.3 \mathrm{~V}$ and $1.3 \mathrm{~V}$, which is peaked at around $1.8 \mathrm{~V}$ (Fig. 1d), accompanied by a significant increase in the optical signal (the region highlighted with green color in Fig. 1c). This process corresponds to the formation of a thin LiF-rich inorganic layer on the $\mathrm{Cu}$ surface that should definitely come from the salt 
anion $\mathrm{PF}_{6}$, and the persistent presence of moisture in electrolyte could be responsible via diversified route ${ }^{41}$, 43.

$$
\begin{gathered}
\mathrm{LiPF}_{6}+\mathrm{H}_{2} \mathrm{O} \rightarrow 2 \mathrm{HF}+\mathrm{POF}_{3}+\mathrm{LiF} \\
\mathrm{HF}+\mathrm{Li}^{+}+e^{-} \rightarrow 0.5 \mathrm{H}_{2}+\mathrm{LiF}
\end{gathered}
$$

The increase of optical responses in this section (Section I) indicates the emergence of a layer that has a smaller refractive index than the electrolyte. Examining all the possible SEI layer's components, we found
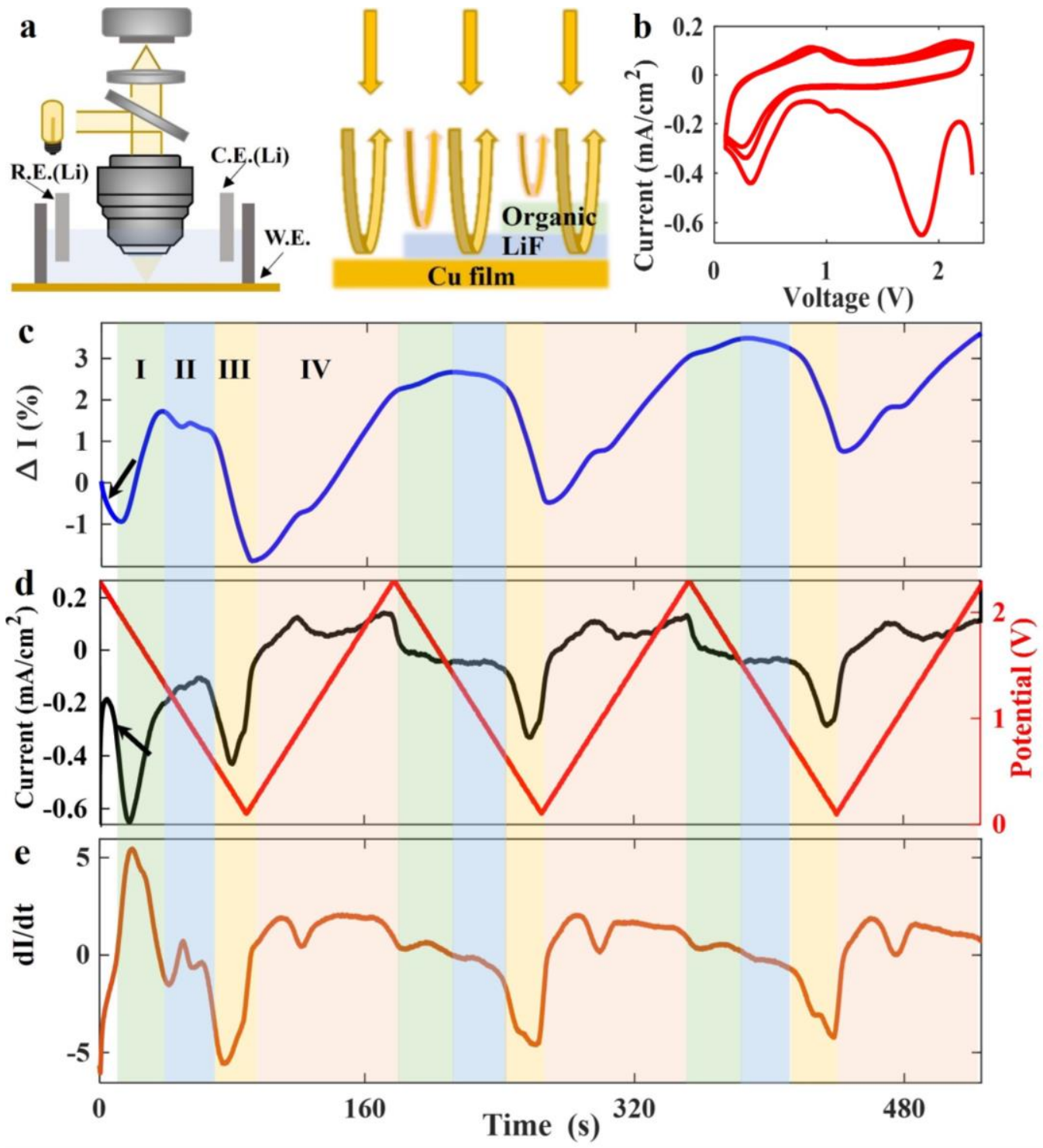

Figure 1 | In-situ characterization of SEI formation dynamics using RIM. a, Schematic diagram of using RIM to image the SEI formation dynamics. b, CV curves of the first three cycles in $1 \mathrm{M} \mathrm{LiPF} / \mathrm{PC}$ with $50 \mathrm{ppm} \mathrm{H}_{2} \mathrm{O}$ as additive. c, The optical reflection signal (RIM signal) during the first three $\mathrm{CV}$ cycles on $\mathrm{Cu}$ electrode in $1 \mathrm{M} \mathrm{LiPF}_{6} / \mathrm{PC}$ with $50 \mathrm{ppm} \mathrm{H}_{2} \mathrm{O}$ additive. I, II, III, and IV are four sections that correspond to LiF-rich layer formation (Section I), EDL formation (Section II), organic-rich SEI deposition (Section III), and re-oxidization (Section IV). d, The corresponding current density (black curve and left axis) and voltage (red curve and right axis) in the first three cycles of CV scans. e, The derivative of optical signals (the curve in c). 
that only LiF fits this description (Supplementary Section 5 and 6). To further validate the observation, a control experiment was conducted using the same electrolyte ( $1 \mathrm{M} \mathrm{LiPF}_{6}$ in PC) without any water additive. We find that the optical responses in the same potential range are much smaller than those in the electrolyte in the presence of $50 \mathrm{ppm}$ water (Supplementary Fig. 4a), indicating a much thinner deposition of LiF-rich layer. Correspondingly, the HF reduction current (Supplementary Fig. 4b) in the CV measurement is also significantly decreased (Supplementary Fig. 5).

In the second Section (Section II with blue highlight in Fig. 1c), a slightly decreased optical signal can be observed. Within this potential window of $1.3 \mathrm{~V}$ to $0.8 \mathrm{~V}$ (Fig. 1b) after the first cycle, the electrolyte mostly remains thermodynamically stable except a minor electrochemical reaction happens at about $1 \mathrm{~V}$ in the first cathodic scan, therefore the change in optical signal should be attributed to the assembly of $\mathrm{Li}^{+}$and the solvent molecules in its solvation sheaths to form an EDL on the deposited LiF-layer. Note that the EDL should exist at the instant the electrode meets an electrolyte, but its structure evolves with both the applied voltage and the new morphology of the electrode surface. In this case, the deposition of LiF renders the electrode surface much rougher as compared with the pristine $\mathrm{Cu}$ surface. This aggregation of solvated cations only changes the structure and composition of EDL at the electrode/electrolyte interface, which is an interfacial behavior of capacitive nature, and has no impact on the permanent chemical composition of the SEI. On the other hand, the accumulation of charges on the electrode surface will change the permittivity of the metal electrode surface and accordingly induce a decrease in the reflected optical intensity. Such EDL-effects have been previously observed by RIM in our previous work ${ }^{44,45}$. Zhou et al. also reported the EDL established at the solid/liquid interface when the $\mathrm{Cu}$ electrode was charged to $1 \mathrm{~V}^{33}$. Furthermore, cointercalation of solvated $\mathrm{Li}^{+}$was also observed when the graphite electrode was discharged to $\sim 0.88 \mathrm{~V}^{39}$.

\section{Reversible emergence and disappearance of organic-rich layer in SEI}

When the potential is lower than $0.8 \mathrm{~V}$, the optical signal decreases significantly (Section III with yellow highlight in Fig. 1c). Correspondingly, the reduction current starts to increase quickly and is peaked at $0.3 \mathrm{~V}$. This corresponds to the reduction of carbonate solvents via a one-electron reduction process as described by equations 3 and 4 to generate $\mathrm{Li}_{2} \mathrm{CO}_{3}$ and $\mathrm{Li}$ alkyl carbonate ${ }^{17}$. The latter might be the priority pathway according to literature ${ }^{39,46}$.

$$
\begin{gathered}
\mathrm{C}_{4} \mathrm{H}_{6} \mathrm{O}_{3}+2 \mathrm{Li}^{+}+2 e^{-} \rightarrow \mathrm{Li}_{2} \mathrm{CO}_{3}+\mathrm{C}_{3} \mathrm{H}_{6} \\
2 \mathrm{C}_{4} \mathrm{H}_{6} \mathrm{O}_{3}+2 \mathrm{Li}^{+}+2 e^{-} \rightarrow \mathrm{C}_{3} \mathrm{H}_{6}\left(\mathrm{OCO}_{2} \mathrm{Li}\right)_{2}+\mathrm{C}_{3} \mathrm{H}_{6}
\end{gathered}
$$

As a result, the main composition of the reduced species is the organic component Li propylene dicarbonate (LPDC) from the $\mathrm{LiPF}_{6} / \mathrm{PC}$ electrolyte, which constitutes an additional organic-rich SEI layer over the inorganic LiF-rich layer on the electrode surface ${ }^{41}$. Since the refractive index of the organic component is much higher than that of the electrolyte (Supplementary Section 5 and 6), the decrease in the optical signal also indicates that the deposited layer in this potential range $(0.8 \mathrm{~V}$ to $0.1 \mathrm{~V})$ is mainly enriched in organic species. When the scan direction is reversed from $0.1 \mathrm{~V}$ (i.e. anodic scan), the obtained optical signal continues to decrease, well in accordance with the current instead of the scan direction. This serves as a clear indication that the cathodic current in this region arises from the electrochemical decomposition of the electrolyte instead of the capacitive behavior associated with the EDL.

In Section IV (highlighted with light purple in Fig. 1c), the electrochemical current starts to turn positive when the potential is higher than $0.25 \mathrm{~V}$, with an anodic event at around $0.9 \mathrm{~V}$. This indicates the reverse of electrochemical reaction from reduction to oxidation on the electrode surface, and the accumulated organic-rich layer in the SEI is partially oxidized through the equation $(5)^{47,48}$.

$$
\mathrm{C}_{3} \mathrm{H}_{6}\left(\mathrm{OCO}_{2} \mathrm{Li}\right)_{2}-2 e^{-} \rightarrow \mathrm{Li}_{2} \mathrm{O}+2 \mathrm{CO}_{2}+\mathrm{C}_{3} \mathrm{H}_{6}+0.5 \mathrm{O}_{2}
$$


The reversible emergence and disappearance of such nascent organic layers has been observed before with other techniques on graphitic surface, such as AFM when an EC-based electrolyte was used ${ }^{49}$, or EQCM when a mixed carbonate electrolyte (EC/DMC) was used ${ }^{39}$, and it has only been observed during the initial cycles of the cell, and the oxidation seems to be partial only, i.e., this organic layer cannot be completely removed. When the potential reaches a more positive region, the current and the differential of optical response (Figs. 1c \& 1e, see discussion in Methods) are both peaked at around $0.9 \mathrm{~V}$. The optical signal shows a significant increase in Section IV (a light purple region in Fig. 1c) during the oxidation process. This is due to the partial oxidation of the organic-rich layer which leads to a decrease of overall SEI presence on the electrode surface. There are also other non-electrochemical and parasitic reactions occurring simultaneously to consume the organic species in the SEI layer and cause the optical signal increase. For example, the HF generated from the hydrolysis of the salt anion $\left(\mathrm{PF}_{6}^{-}\right)$will eliminate alkyl carbonate via equation (6) ${ }^{50}$. The produced $\mathrm{Li}_{2} \mathrm{CO}_{3}$ (from equation (3)) can also react with salt in the electrolyte via equation $(7)^{51}$.

$$
\begin{aligned}
& \mathrm{C}_{3} \mathrm{H}_{6}\left(\mathrm{OCO}_{2} \mathrm{Li}\right)_{2}+2 \mathrm{HF} \rightarrow 2 \mathrm{LiF}+2 \mathrm{CO}_{2}+\mathrm{C}_{3} \mathrm{H}_{6}(\mathrm{OH})_{2} \\
& \mathrm{LiPF}_{6}+2 \mathrm{Li}_{2} \mathrm{CO}_{3} \rightarrow 4 \mathrm{LiF}+2 \mathrm{CO}_{2}+\mathrm{F}_{2} \mathrm{PO}_{2} \mathrm{Li}
\end{aligned}
$$

These two possible chemical reaction pathways help account for the increase in the optical signal.

\section{Scan-hold-release experiment}

To further verify observations obtained in the CV scans, a scan-hold-release experiment was performed. The potential was initially scanned from $2.3 \mathrm{~V}$ to different pre-selected cut-off potentials of $1.0 \mathrm{~V}, 0.6 \mathrm{~V}$, $0.3 \mathrm{~V}$, and $0.1 \mathrm{~V}$, subsequently held at these levels for $100 \mathrm{~s}$, and finally released. The optical responses were recorded in Fig. 2a. At the early stage of the scan (from $2.3 \mathrm{~V}$ to $1.3 \mathrm{~V}$ ), the increase of the optical reflection signals was apparent (highlighted with green color in Fig. 2a). This is correlated to the LiF formation on the electrode surface (correlated to Section I in Fig.1c). When the scan continued from $1.3 \mathrm{~V}$ to $0.8 \mathrm{~V}$, the optical signal decreased, which corresponds to the EDL assembly (highlighted with blue color in Fig. 2a and correlated to Section II in Fig. 1c). When the potential was held at $1.0 \mathrm{~V}$ where there is no significant electrochemical reaction happening in this voltage range (from $1.3 \mathrm{~V}$ to $0.8 \mathrm{~V}$ ), a flat line in the optical response was observed (black curve in Fig. 2a). When the potential was further decreased down to $0.6 \mathrm{~V}$, a significant decrease of optical reflectance occurred (blue curve in Fig. 2a), which indicates the formation of organic-rich layer in SEI (highlighted with yellow color in Fig. 2a and Section III). In addition, holding the potential at $0.6 \mathrm{~V}$ for $100 \mathrm{~s}$ led to a continuous decrease in the optical signal, indicating that the reduction continues and the organic-rich SEI layer keeps growing. For the $0.3 \mathrm{~V}$ (brown curve in Fig. 2a)
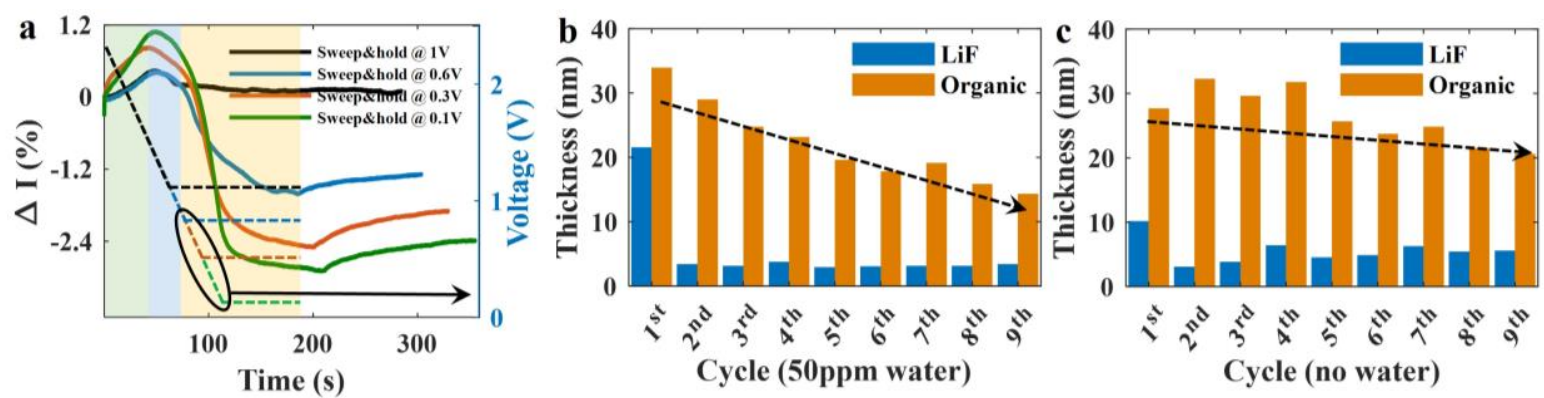

Figure 2 | The growth and evolution of SEI layers. a, Scan-hold-release experiment. The solid lines are RIM signals at different conditions, and the dashed lines are the corresponded potential (to the right axis). b. The thickness accumulation of the LiF-rich layer (blue bar) and the organic-rich layer (Orange bar) in each $\mathrm{CV}$ cycle in $1 \mathrm{M} \mathrm{LiPF}_{6} / \mathrm{PC}$ with $50 \mathrm{ppm} \mathrm{H}_{2} \mathrm{O}$ additive. c, The thickness accumulation of the LiF-rich layer (blue bar) and the organic-rich layer (Orange bar) in each CV cycle in $1 \mathrm{M} \mathrm{LiPF} / \mathrm{PC}$ without $\mathrm{H}_{2} \mathrm{O}$ additive. 
and $0.1 \mathrm{~V}$ (green curve in Fig. 2a), the signal decreases in Section III are much more significant than that of $0.6 \mathrm{~V}$. This is because a larger amount of organic-rich SEI was formed on the electrode. When we remove the applied potentials $(0.6 \mathrm{~V}, 0.3 \mathrm{~V}$, and $0.1 \mathrm{~V})$, a portion of ions in the EDL will be released from the interface, which is the reason why the optical reflectance increases initially. Furthermore, all three experiments $(0.6 \mathrm{~V}, 0.3 \mathrm{~V}$, and $0.1 \mathrm{~V})$ have much lower optical signal after the signal is stabilized, which indicates the organic-rich outer layer still remains on the electrode surface and it will not diffuse away or decompose automatically. These experiments provide strong evidence for our observation of the dynamic formation process of SEI layers observed in the CV scans.

\section{Growth and evolution of SEI}

To understand the evolution and maturation process of SEI over cycling, we performed the CV scans for 9 cycles continuously and recorded the optical reflection signals. For each cycle, the thicknesses of LiFrich inner and the organic-rich outer layers of the SEI were extracted from the optical reflection signal changes (see more details in Methods). The results for the electrolytes of $1 \mathrm{M} \mathrm{LiPF}_{6} / \mathrm{PC}$ in the absence or presence of $50 \mathrm{ppm}$ water are summarized in Figs. $2 \mathrm{~b}$ and 2c, respectively. Water promotes a thicker LiFrich SEI layer ( $23 \mathrm{~nm}$, Fig. $2 b)$ in the first cycle, which was signified by a much higher signal increase in Section I in Fig. 1c (green color), and is apparently benefitted from the generation and subsequent of HF (equation 2). After the first cycle, the LiF-rich SEI layer continues to accumulate on the electrode surface but at a much slower rate $(\sim 3.5 \mathrm{~nm} /$ cycle, Fig. $2 b)$, because the thick layer of LiF formed in the first cycle minimizes the further charge-transfer across the interface.

In comparison, the electrolyte without additional water shows quite different behaviors. In the first cycle, a much thinner LiF-rich layer $(\sim 10 \mathrm{~nm})$ is deposited onto the $\mathrm{Cu}$ electrode surface, proving the correlation between water content and the origin of more LiF deposition. Note that trace water ( $20 \mathrm{ppm})$ is always present in liquid electrolytes even after the solvents are rigorously dried ${ }^{40}$. The deposition rate of $\mathrm{LiF}$ layer after the first cycle in the electrolyte without water additive remains higher $(\sim 5.0 \mathrm{~nm} /$ cycle, Fig. 2c) than that with $50 \mathrm{ppm}$ water additive $(\sim 3.5 \mathrm{~nm} / \mathrm{cycle}$, Fig. $2 \mathrm{~b})$. This indicates that the quality of such LiF-rich SEI layer formed in the absence of water is relatively less effective in insulating electron, which requires more $\mathrm{LiF}$ deposition on the electrode in each of the following cycles. Meanwhile, all first three cycles share a similar EDL formation signal (Fig. 1c, Section II, highlighted with blue color), indicating the difference in SEI quality does not affect the charge assembly on the electrode/SEI layer, which is essentially a capacitive-like behavior and depends little on the SEI chemistry.

The organic-rich layer in SEI is another important characteristic that determines overall electrolyte consumption in the irreversible and parasitic processes. We extracted the organic-rich layer's thickness formed in each cycle and plotted them in Figs. 2b-c. Note that after deposition of the organic-rich layer in the first half of the cycle, it will be mostly removed by the re-oxidation process. In presence of $50 \mathrm{ppm}$ water, the organic-rich SEI layer deposited in the first cycle is around $35 \mathrm{~nm}$. As more LiF is deposited onto the electrode surface, the thickness of organic-rich deposition layer in each cycle quickly decreases (Fig. 2b). On the other hand, in the absence of $50 \mathrm{ppm}$ water, this thickness is relatively stable over cycles (Fig. 2c). This observation directly links water content to SEI quality and irreversible electrolyte consumption.

\section{Spatial correlations between the LiF-rich and the organic layers in SEI}

Note that the optical reflectance curves in Figs. $1 \& 2$ are averaged from the entire observation window ( $80 \mu \mathrm{m}$ by $100 \mu \mathrm{m}$ ), however, the local heterogeneity is smoothed out. Using RIM, we mapped the morphologies of LiF-rich and organic-rich layers in the SEI at three different potentials, as shown in Figs. 3a-f. At $2.0 \mathrm{~V}, \mathrm{LiF}$ begins to grow on the $\mathrm{Cu}$ electrode surface with an averaged thickness of $\sim 5 \mathrm{~nm}$ and a surface roughness of $\sim 0.5 \mathrm{~nm}$ (standard deviation, Fig. 3h). After the initial deposition, the LiF-rich layer continues to grow in thickness to $\sim 12 \mathrm{~nm}$ at $1.7 \mathrm{~V}$ (Fig. 3b), and $\sim 23 \mathrm{~nm}$ at $1.4 \mathrm{~V}$ (Fig. 3c). Correspondingly, the surface roughness also increases to $0.7 \mathrm{~nm}$ at $1.7 \mathrm{~V}$ and $0.9 \mathrm{~nm}$ at $1.4 \mathrm{~V}$ (Fig. 3h). The organic-rich 

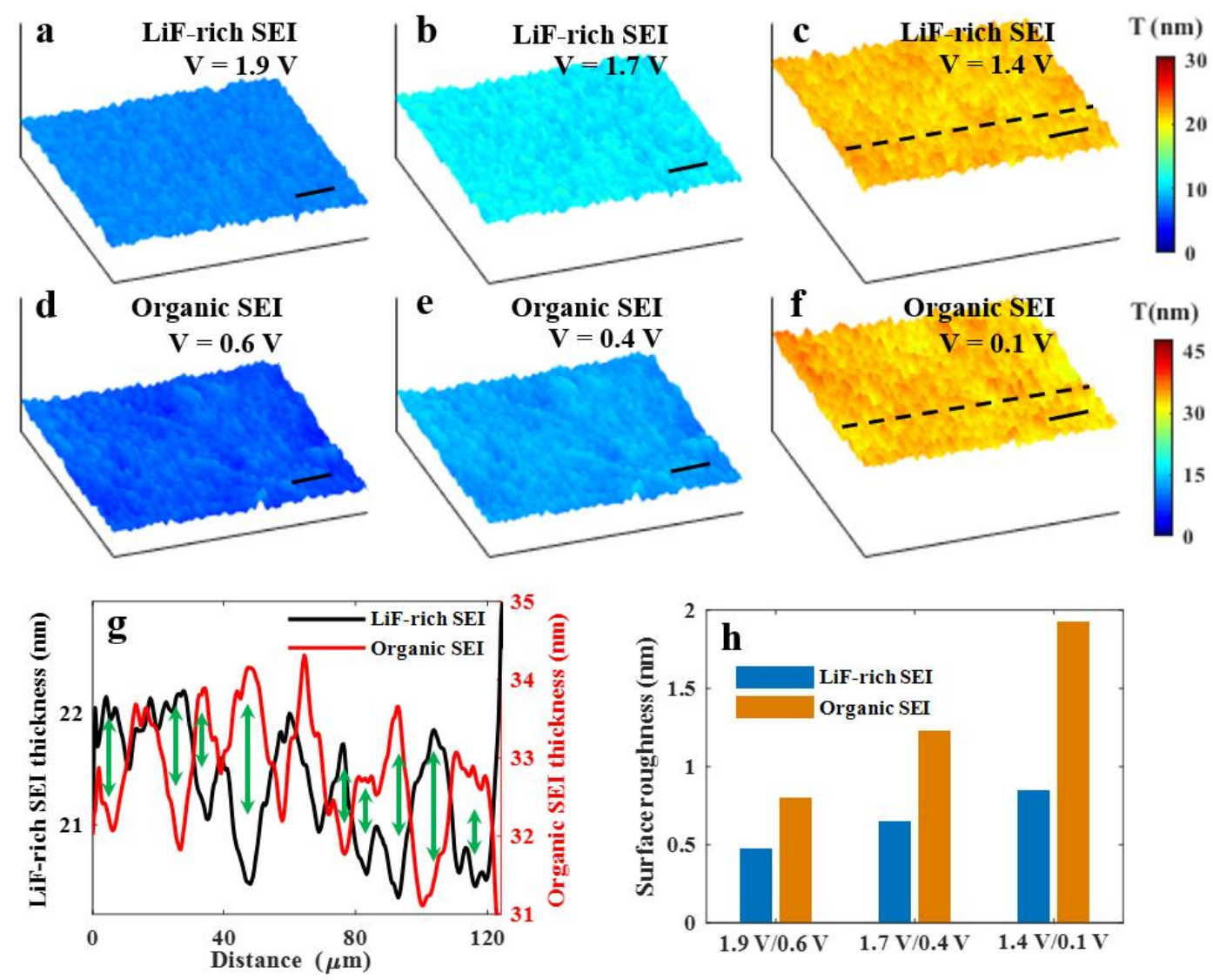

Figure 3 | Image the localized SEI formation dynamics using RIM. a-c, The morphology maps of LiF-rich layer in $1 \mathrm{M} \mathrm{LiPF}_{6} / \mathrm{PC}$ with $50 \mathrm{ppm} \mathrm{H}_{2} \mathrm{O}$ at potentials $\mathrm{V}=1.9 \mathrm{~V}(\mathbf{a}), \mathrm{V}=1.7 \mathrm{~V}(\mathbf{b})$, and $\mathrm{V}=$ $1.4 \mathrm{~V}$ (c). d-f, The morphology maps of organic-rich SEI layer in $1 \mathrm{M} \mathrm{LiPF}_{6} / \mathrm{PC}$ with $50 \mathrm{ppm}_{2} \mathrm{O}$ at potentials $\mathrm{V}=0.6 \mathrm{~V}(\mathbf{d}), \mathrm{V}=0.4 \mathrm{~V}(\mathbf{e})$, and $\mathrm{V}=0.1 \mathrm{~V}(\mathbf{f})$. g, The thickness profiles of LiF-rich layer (left axis and black line) and organic-rich layer (right axis and red line) across the same line. The profile lines have been shown in Figs. $3 \mathbf{c} \& \mathbf{f}$ as the dashed black line. h, The surface roughness of LiF-rich and organic-rich layers shown in a-f. Scale bar: $20 \mu \mathrm{m}$.

layer's growth starts from $0.8 \mathrm{~V}$ (Fig. 3d). Both the average thickness and the surface roughness quickly increase as the potential decreases (Figs. 3d-f and 3h), and the thickness of the organic layer in the SEI reaches as high as $\sim 35 \mathrm{~nm}$.

The deposition of LiF-rich layer will significantly influence the surface reactions, including the growth of the organic-rich layer. To understand the correlation between these two layers, we plotted the height profiles of LiF-rich layer and organic-rich layer across the same line (along black dashed lines in Figs. 3c\&f) in Fig. 3g. A strong inverse spatial correlation exists at the locations that the LiF-rich layer is thin while the organic layer is relatively thick, and vise versa. To further illustrate such correlations, we plotted the thickness maps of these two layers (Fig. 4), separately. The region circled by the black dashed line has thinner LiF-rich inorganic layer (Fig. 4a) and thicker organic-rich SEI layer (Fig. 4b-c). The regions circled by the red solid line have a thicker LiF-rich inorganic layer (Fig. 4a) and thinner organic-rich SEI layer (Figs. 4b-c). The inverse relation is more pronounced when we compare the smaller electrode areas. The red and black arrows correspond to the regions that have thicker and thinner LiF-rich inorganic SEI layers, respectively (Fig. 4a), and the corresponding regions in organic-rich layer maps (Figs. 4b-c) show opposite responses. 


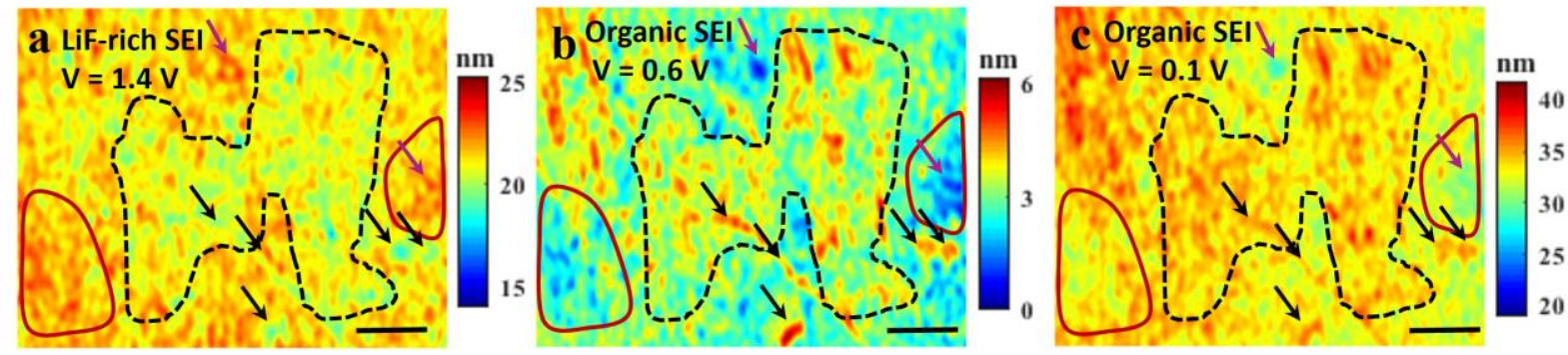

Figure 4 | The spatial correlation between the LiF-rich layer and the organic layer in SEI. a, The LiF-rich layer morphology at $\mathrm{V}=1.4 \mathrm{~V}$. Black dashed circle: thin LiF-rich layer region, red solid circle: thick LiF-rich layer regions, purple arrows: thick LiF-rich layer locations, black arrows: thin LiF-rich layer locations. b-c, Organic-rich SEI morphology at early stage of deposition $(\mathrm{V}=0.6 \mathrm{~V}$, b) and late stage of deposition $(\mathrm{V}=0.1 \mathrm{~V}, \mathbf{c})$. Black dashed circle, red solid circles, purple arrows, and black arrows indicate the same regions or locations with a. Scale bar: $20 \mu \mathrm{m}$.

It is widely accepted that the inorganic layer (mainly LiF-rich) in SEI serves as the key component that blocks the electron transfer and allows Li ion to diffuse through it. However, depending on the quality and thickness of the LiF-rich layer, a small number of electrons can still tunnel through the LiF layer and induce the parasitic reactions, leading to the progressive growth of the organic-rich outer layer. A thicker LiF layer will block electrons more effectively and result in a thinner organic-rich outer layer, while a thinner $\mathrm{LiF}$ layer will lead to a less efficient blocking of electrons and a thicker organic-rich layer.

\section{SEI effects on Li nucleation}

The heterogeneity in both chemical composition and morphology of the SEI directly affects Li nucleation and growth, which are closely related to the performance of all Li-based battery chemistries. By polarizing the $\mathrm{Cu}$ electrode further down to lower potentials, we induced $\mathrm{Li}^{0}$-deposition while applying RIM to correlate the SEI quality and the subsequent Li nucleation dynamics in the absence or presence of the water additive. Galvanostatic electrodeposition was applied to form the SEI and induce Li nucleation. Figs. 5a and 5b show the optical (top figures) and the corresponding voltage signal (bottom figures) during these processes in the presence (Fig. 5a) and absence (Fig. 5b) of an additional 50 ppm water additive. The Sections I III can be respectively identified, which corresponds well to our previous CV experiments, and verifies that SEI layers formed in either galvanostatic or potentiodynamic (CV) manner generate similar optical responses under RIM, hence confirming the universal reliability of RIM. The thicknesses of LiFrich and organic-rich layers were also extracted (Fig. 5c), which are much thicker due to the extra enforcement force from the applied constant current (Supplementary Section 12).

When the potential of the working electrode goes below $0 \mathrm{~V}$, the optical signals start to decrease (pointed by the black arrows in Figs. 5a-b), due to the emergence of light-scattering $\mathrm{Li}^{0}$ nuclei. Figs. $5 \mathrm{f}$ and 5 i show the optical intensity map of Li nucleation. (Fig. 5 f corresponds to the snapshot image at $128 \mathrm{~s}$ and Fig. 5i corresponds to the snapshot of nucleation at $180 \mathrm{~s}$ ). For better visualization, we reverse the optical response so that the brighter intensity represents more $\mathrm{Li}^{0}$ nucleation in Figs. $5 \mathrm{f}$ and $5 \mathrm{i}$. It becomes apparent that the $\mathrm{Li}^{0}$ nucleation is not uniform in the electrolyte without water additive (Fig. $5 \mathrm{f}$ ). However, the uniformity is significantly improved with the introduction of $50 \mathrm{ppm}$ water (Fig. 5i). By comparing LiFrich layer thickness maps (Figs. 5d and 5g) and organic layer thickness maps (Figs. 5e and 5h), it can be inferred that the electrolyte with $50 \mathrm{ppm}$ water additive leads to both the LiF layer and the organic layer to be much more uniform, which facilitates a more even distribution of Li nuclei. 

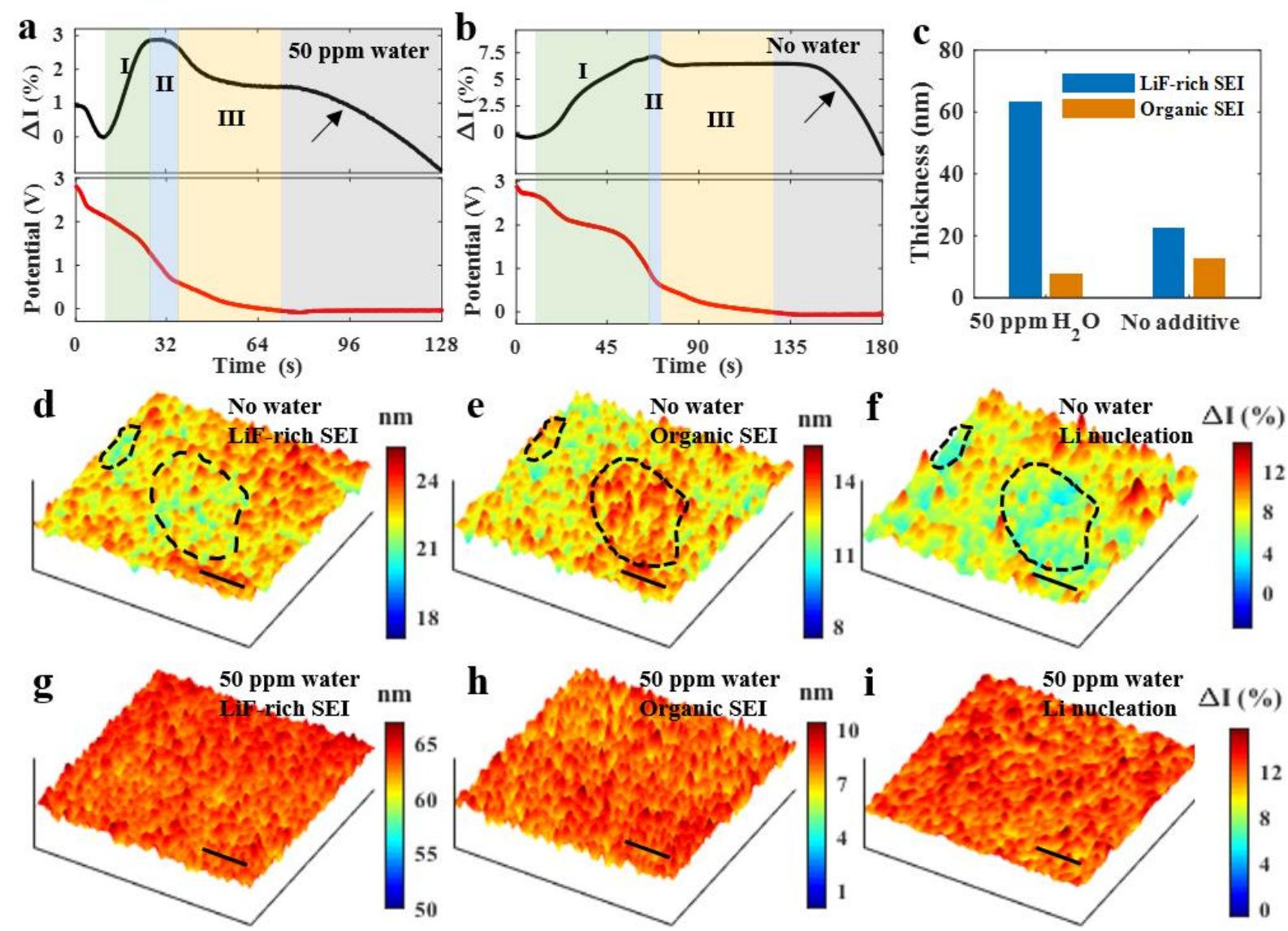

Figure 5 | SEI effects on Li nucleation. a-b, The constant current measurement in $1 \mathrm{M} \mathrm{LiPF}_{6} / \mathrm{PC}$ with $50 \mathrm{ppm} \mathrm{H}_{2} \mathrm{O}(\mathbf{a})$, and without water additive (b). The experiment was performed with constant current at $0.1 \mathrm{~mA} \mathrm{~cm}^{-2}$ (voltages at end of deposition are $40 \mathrm{mV}$ and $42 \mathrm{mV}$ correspondingly). The top figures are the optical reflection responses during the measurement, and the bottom figures are the corresponding potentials. I, II, and III correspond to the Sections I, II and III, respectively (see manuscript for more details). c, The thicknesses of LiF-rich and organic-rich layers are extracted from the experiments in a and b. d-f, The morphology maps of LiF-rich SEI layer (d) and organic-rich SEI layer (e) in the electrolyte without water additive, and $\mathbf{f}$ shows the optical response of Li nucleation in the electrolyte without water additive. g-i, The morphology maps of LiF-rich SEI layer (g) and organicrich SEI layer (h) in the electrolyte with $50 \mathrm{ppm}$ water additive, and $\mathbf{i}$ shows the optical response of Li nucleation in the electrolyte with $50 \mathrm{ppm}$ water additive. Note that $\Delta \mathrm{I}$ in $\mathbf{f}$ and $\mathbf{i}$ are the intensity change caused by the Li nucleation and the bigger $\Delta \mathrm{I}$ means more Li nucleation. Scale bar: $20 \mu \mathrm{m}$.

\section{Conclusion}

This work develops an in-operando RIM to map the minimal localized SEI information. The optical interference amplifies the signal and allows us to probe the localized responses from the stratified SEI structure and its evolution during cell cycling. RIM also provides a unique capability of quantitatively differentiating the signals generated from the LiF-rich and organic-rich SEI sub-components, thus generating a reliable descriptor to evaluate the SEI quality. Equipped with this powerful tool, we identified the strong inverse correlation between the LiF-rich inner layer and the organic-rich outer layer, while trace water presence $(50 \mathrm{ppm})$ promotes the former and suppresses the latter. The inorganic layer serves as the 
key sub-component to homogenize the Li nucleation distribution. The fundamental understanding provides powerful guideline to design better electrolytes and interphases for high-performance LMBs.

\section{Methods}

In-operando RIM. The in-operando RIM was built on an upright Olympus BX50 microscope in an argonfilled glovebox (MBraun, with $\mathrm{H}_{2} \mathrm{O}$ and $\mathrm{O}_{2}$ level less than $0.5 \mathrm{ppm}$ ). The electrolytes have relatively high refractive index (around 1.415 in our case) comparing with that of air $(\sim 1)$, and this refractive index mismatch will lead to aberrations that blurs the images. To minimize the refractive index mismatch influence, a special $20 \mathrm{X}$ multi-immersion objective (Applied Scientific Instrumentation) was used. The objective can provide refractive index match in a wide range from 1.33 to 1.56 , which completely meets the imaging requirements in the electrolytes that are commonly used in LMBs and LIBs at present. The numerical aperture of the objective is around 0.68 in the electrolyte that has a refractive index of 1.415 . The incident light is mainly distributed around $600 \mathrm{~nm}$. The spatial resolution of the RIM is defined by the diffraction limit $(\sim 300 \mathrm{~nm})$ and the temporal resolution is defined by our camera framerate. To catch the electrochemical reaction process, we chose the frame rate of $\sim 10$ frame per second in this research, but it can be ramped up to 1,000 frame per second if necessary. The observation window was around $230 \mu \mathrm{m} \times$ $260 \mu \mathrm{m}$ which was determined by a charge-coupled device (CCD) camera (Pike, F032C monochrome). During the experiment, the dynamic change in SEI will induce the interference and affect the reflected light intensity, which is recorded with the CCD camera. The recorded images are synchronized with the applied electrochemical potentials using a Labview card (Supplementary Section 3). The camera was connected to a computer outside the glovebox with the feedthrough cable, and the camera and the potentiostat were synchronized and controlled by the same computer. The CCD received reflection optical intensity is the result of light interferences that happen between different layers on the substrate, which is closely related to the refractive index of the dielectric medium. When layers with different composition form on the electrode substrate, the reflection optical signal will increase or decrease according to the refractive index change and is very sensitive the thickness of the formed layer.

Differential of optical response. When the electrochemical reactions happen on the electrode, the chemical composition and morphology of the surface change, and the optical reflectance signal reflect such overall changes. On the other hand, the integration of electrochemical current quantifies the total reactions that happens on the surface via coulomb counts. Therefore, a mathematic relation should exist between the current and the derivative of the optical signal, which signifies each of the electrochemical reactions that happen. Such relation is revealed in Fig. 1e, where the derivative of optical response displays "finger-print" match with the electrochemical current (red curve in Fig. 1d). This serves as the strong quantitative evidence to the argument that what optically detected does reflect the electrochemical reactions that happen on the electrode surface.

The relationship between optical reflectance and refractive index. When the light is reflected from different surfaces of a thin film, the interference will happen, and a phase difference will be introduced to influence the detected optical reflectance intensity. Depending on the refractive index difference between the surrounding environment and the newly formed layer, different degrees of constructive interference or destructive interference will occur. Thus, through detecting the reflected intensity change, we can easily recognize the characteristics of the component that is deposited on the substrate. In our system, the refractive index of the bulk electrolyte is 1.415. And when the LiF-rich SEI (refractive index is 1.32) accumulates on the substrate, we will observe an increasing optical signal. On the other hand, when the organic-rich SEI (refractive index is around 1.46) forms, there will be a decreasing optical signal. Details are provided in the Supplementary Section 4 and 6. 
Converting reflectance to thickness. Based on Fresnel equations, the Winspall software package is used to simulate the reflectivity response from multilayer interfaces. A dual-layer model of the SEI is adopted in the simulation since it conforms the already reported conclusions and satisfies the optical response in our experiments. The parameters for simulation are summarized in Supplementary Table 1. The simulation results in Supplementary Fig. 2 elucidate how the thin film thickness change will influence the reflectivity. Then, according to the detected optical signal change we can obtain the thickness information of the formed layer for further analysis. More details and discussions are provided in Supplementary Section 6.

Morphology analysis. The morphology and thickness of the SEI layers are obtained through the calibration curves established from the experiments shown in Supplementary Figs. 2. We have synchronized the optical images with the applied electrochemical potentials (see more discussions in Supplementary Section 3), each frame of the images can be correlated to the specific SEI formation dynamic process. For example, the total amount of reflectance change caused by the LiF-rich SEI is calculated by subtracting the initial reflection image (first frame of the entire sequence) from the last frame at the end of Section I. Then converting the intensity value of each pixel to thickness using the calibration curves and replot the image. The morphology and localized information of the LiF-rich SEI are thereby achieved. Similarly, the difference between the last frame and the first frame in Section III represents the reflectance changes caused by the organic-rich SEI formation on the electrode surface.

\section{Acknowledgements}

This work was supported by the start-up fund at the University of Houston, the University of Houston's University of Houston Center for Carbon Management in Energy program, support from Beyond Bits Technology, Inc., and University Training and Research for Fossil Energy Applications (DOE DE-FE0032092) (X.S.). The work performed at Pacific Northwest National Laboratory (PNNL) was supported by the Assistant Secretary for Energy Efficiency and Renewable Energy, Vehicle Technologies Office, of the U.S. Department of Energy (DOE) through the Advanced Battery Materials Research (BMR) program under contract number DE-AC05-76RL01830. PNNL is operated by Battelle for the U.S. DOE under Contract DE-AC05-76RL01830. Y.Y. acknowledges the funding support from UH Advanced Manufacturing Institute. 


\section{Reference}

1. Goodenough JB, Park KS. The Li-Ion Rechargeable Battery: A Perspective. Journal of the American Chemical Society 2013, 135(4): 1167-1176.

2. Dunn B, Kamath H, Tarascon JM. Electrical Energy Storage for the Grid: A Battery of Choices. Science 2011, 334(6058): 928-935.

3. Grande L, Paillard E, Hassoun J, Park JB, Lee YJ, Sun YK, et al. The Lithium/Air Battery: Still an Emerging System or a Practical Reality? Advanced Materials 2015, 27(5): 784-800.

4. Lu YY, Tikekar M, Mohanty R, Hendrickson K, Ma L, Archer LA. Stable Cycling of Lithium Metal Batteries Using High Transference Number Electrolytes. Adv Energy Mater 2015, 5(9): 7.

5. Arico AS, Bruce P, Scrosati B, Tarascon JM, Van Schalkwijk W. Nanostructured materials for advanced energy conversion and storage devices. Nat Mater 2005, 4(5): 366-377.

6. Sun YM, Liu NA, Cui Y. Promises and challenges of nanomaterials for lithium-based rechargeable batteries. Nat Energy 2016, 1: 12.

7. Cheng XB, Zhang Q. Dendrite-free lithium metal anodes: stable solid electrolyte interphases for high-efficiency batteries. J Mater Chem A 2015, 3(14): 7207-7209.

8. Tarascon JM, Armand M. Issues and challenges facing rechargeable lithium batteries. Nature 2001, 414(6861): 359-367.

9. Guo YP, Li HQ, Zhai TY. Reviving Lithium-Metal Anodes for Next-Generation High-Energy Batteries. Advanced Materials 2017, 29(29): 25.

10. Yuan SY, Bao JL, Wang LN, Xia YY, Truhlar DG, Wang YG. Graphene-Supported Nitrogen and Boron Rich Carbon Layer for Improved Performance of Lithium-Sulfur Batteries Due to Enhanced Chemisorption of Lithium Polysulfides. Adv Energy Mater 2016, 6(5): 9.

11. Janek J, Zeier WG. A solid future for battery development. Nat Energy 2016, 1: 4.

12. Luntz AC, Voss J, Reuter K. Interfacial Challenges in Solid-State Li lon Batteries. J Phys Chem Lett 2015, 6(22): 4599-4604.

13. Cao RG, Xu W, Lv DP, Xiao J, Zhang JG. Anodes for Rechargeable Lithium-Sulfur Batteries. Adv Energy Mater 2015, 5(16): 23.

14. He P, Zhang T, Jiang J, Zhou HS. Lithium-Air Batteries with Hybrid Electrolytes. J Phys Chem Lett 2016, 7(7): 1267-1280.

15. Huang C-J, Thirumalraj B, Tao H-C, Shitaw KN, Sutiono H, Hagos TT, et al. Decoupling the origins of irreversible coulombic efficiency in anode-free lithium metal batteries. Nat Commun 2021, 12(1): 1-10.

16. Qiao Y, Yang H, Chang Z, Deng H, Li X, Zhou H. A high-energy-density and long-life initial-anodefree lithium battery enabled by a Li2O sacrificial agent. Nat Energy 2021, 6(6): 653-662.

17. Xu K. Nonaqueous liquid electrolytes for lithium-based rechargeable batteries. Chem Rev 2004, 104(10): 4303-4417.

18. Peled $\mathrm{E}$. The electrochemical behavior of alkali and alkaline earth metals in nonaqueous battery systems - the solid electrolyte interphase model. J Electrochem Soc 1979, 126(12): 2047.

19. Gauthier M, Carney TJ, Grimaud A, Giordano L, Pour N, Chang H-H, et al. Electrode-electrolyte interface in Li-ion batteries: current understanding and new insights. The journal of physical chemistry letters 2015, 6(22): 4653-4672.

20. An SJ, Li J, Daniel C, Mohanty D, Nagpure S, Wood III DL. The state of understanding of the lithium-ion-battery graphite solid electrolyte interphase (SEI) and its relationship to formation cycling. Carbon 2016, 105: 52-76.

21. Orsini F, Du Pasquier A, Beaudoin B, Tarascon J, Trentin M, Langenhuizen N, et al. In situ scanning electron microscopy (SEM) observation of interfaces within plastic lithium batteries. $J$ Power Sources 1998, 76(1): 19-29. 
22. Mehdi BL, Qian J, Nasybulin E, Park C, Welch DA, Faller R, et al. Observation and Quantification of Nanoscale Processes in Lithium Batteries by Operando Electrochemical (S)TEM. Nano Lett 2015, 15(3): 2168-2173.

23. Nie MY, Chalasani D, Abraham DP, Chen YJ, Bose A, Lucht BL. Lithium Ion Battery Graphite Solid Electrolyte Interphase Revealed by Microscopy and Spectroscopy. Journal of Physical Chemistry C 2013, 117(3): 1257-1267.

24. Wang L, Menakath A, Han F, Wang Y, Zavalij PY, Gaskell KJ, et al. Identifying the components of the solid-electrolyte interphase in Li-ion batteries. Nature chemistry 2019, 11(9): 789-796.

25. Ai Q, Fang Q, Liang J, Xu X, Zhai T, Gao G, et al. Lithium-conducting covalent-organic-frameworks as artificial solid-electrolyte-interphase on silicon anode for high performance lithium ion batteries. Nano Energy 2020, 72: 104657.

26. Zeng X, Mao J, Hao J, Liu J, Liu S, Wang Z, et al. Electrolyte Design for In Situ Construction of Highly Zn2+ - Conductive Solid Electrolyte Interphase to Enable High - Performance Aqueous Zn - Ion Batteries under Practical Conditions. Advanced Materials 2021, 33(11): 2007416.

27. Nanda J, Yang G, Hou T, Voylov DN, Li X, Ruther RE, et al. Unraveling the nanoscale heterogeneity of solid electrolyte interphase using tip-enhanced Raman spectroscopy. Joule 2019, 3(8): 2001-2019.

28. Chen D, Feng C, Han Y, Yu B, Chen W, Zhou Z, et al. Origin of extra capacity in the solid electrolyte interphase near high-capacity iron carbide anodes for Li ion batteries. Energy \& Environmental Science 2020, 13(9): 2924-2937.

29. Qiu H, Du X, Zhao J, Wang Y, Ju J, Chen Z, et al. Zinc anode-compatible in-situ solid electrolyte interphase via cation solvation modulation. Nat Commun 2019, 10(1): 1-12.

30. Wood KN, Steirer KX, Hafner SE, Ban C, Santhanagopalan S, Lee S-H, et al. Operando X-ray photoelectron spectroscopy of solid electrolyte interphase formation and evolution in Li2S-P2S5 solid-state electrolytes. Nat Commun 2018, 9(1): 1-10.

31. Cheng D, Wynn TA, Wang X, Wang S, Zhang M, Shimizu R, et al. Unveiling the stable nature of the solid electrolyte interphase between lithium metal and LiPON via cryogenic electron microscopy. Joule 2020, 4(11): 2484-2500.

32. Cao C, Abate II, Sivonxay E, Shyam B, Jia C, Moritz B, et al. Solid electrolyte interphase on native oxide-terminated silicon anodes for Li-ion batteries. Joule 2019, 3(3): 762-781.

33. Zhou Y, Su M, Yu X, Zhang Y, Wang J-G, Ren X, et al. Real-time mass spectrometric characterization of the solid-electrolyte interphase of a lithium-ion battery. Nature nanotechnology 2020, 15(3): 224-230.

34. Li YZ, Li YB, Pei AL, Yan K, Sun YM, Wu CL, et al. Atomic structure of sensitive battery materials and Interfaces revealed by cryo-electron microscopy. Science 2017, 358(6362): 506-510.

35. Wang XF, Zhang MH, Alvarado J, Wang S, Sina M, Lu BY, et al. New Insights on the Structure of Electrochemically Deposited Lithium Metal and Its Solid Electrolyte Interphases via Cryogenic TEM. Nano Lett 2017, 17(12): 7606-7612.

36. Wan J, Hao Y, Shi Y, Song Y-X, Yan H-J, Zheng J, et al. Ultra-thin solid electrolyte interphase evolution and wrinkling processes in molybdenum disulfide-based lithium-ion batteries. Nat Commun 2019, 10(1): 1-10.

37. von Cresce A, Russell SM, Baker DR, Gaskell KJ, Xu K. In Situ and Quantitative Characterization of Solid Electrolyte Interphases. Nano Lett 2014, 14(3): 1405-1412.

38. Pathak R, Chen K, Gurung A, Reza KM, Bahrami B, Pokharel J, et al. Fluorinated hybrid solidelectrolyte-interphase for dendrite-free lithium deposition. Nat Commun 2020, 11(1): 1-10.

39. Liu T, Lin L, Bi X, Tian L, Yang K, Liu J, et al. In situ quantification of interphasial chemistry in Liion battery. Nature nanotechnology 2019, 14(1): 50-56. 
40. Nie M, Abraham DP, Seo DM, Chen Y, Bose A, Lucht BL. Role of solution structure in solid electrolyte interphase formation on graphite with LiPF6 in propylene carbonate. The Journal of Physical Chemistry C 2013, 117(48): 25381-25389.

41. Qian J, Xu W, Bhattacharya P, Engelhard M, Henderson WA, Zhang Y, et al. Dendrite-free Li deposition using trace-amounts of water as an electrolyte additive. Nano Energy 2015, 15: 135144.

42. Shen C, Yan H, Gu J, Gao Y, Yang J, Xie K. Li2O-Reinforced solid electrolyte interphase on threedimensional sponges for dendrite-free lithium deposition. Frontiers in chemistry 2018: 517.

43. Terborg L, Nowak S, Passerini S, Winter M, Karst U, Haddad PR, et al. Ion chromatographic determination of hydrolysis products of hexafluorophosphate salts in aqueous solution. Analytica chimica acta 2012, 714: 121-126.

44. Shi Y, Feng G, Li X, Yang X, Ghanim AH, Ruchhoeft $P$, et al. Electrochemical Impedance Imaging on Conductive Surfaces. Anal Chem 2021, 93(36): 12320-12328.

45. Foley KJ, Shan X, Tao NJ. Surface impedance imaging technique. Anal Chem 2008, 80(13): 51465151.

46. Aurbach D, Daroux M, Faguy $P$, Yeager E. Identification of surface films formed on lithium in propylene carbonate solutions. J Electrochem Soc 1987, 134(7): 1611.

47. Xing L, Li W, Wang C, Gu F, Xu M, Tan C, et al. Theoretical investigations on oxidative stability of solvents and oxidative decomposition mechanism of ethylene carbonate for lithium ion battery use. The Journal of Physical Chemistry B 2009, 113(52): 16596-16602.

48. Kanamura K, Umegaki T, Ohashi M, Toriyama S, Shiraishi S, Takehara Z-i. Oxidation of propylene carbonate containing LiBF4 or LiPF6 on LiCoO2 thin film electrode for lithium batteries. Electrochim Acta 2001, 47(3): 433-439.

49. Cresce Av, Russell SM, Baker DR, Gaskell KJ, Xu K. In situ and quantitative characterization of solid electrolyte interphases. Nano Lett 2014, 14(3): 1405-1412.

50. Aurbach D, Zaban A, Schechter A, Ein - Eli Y, Zinigrad E, Markovsky B. The study of electrolyte solutions based on ethylene and diethyl carbonates for rechargeable Li batteries: I. Li metal anodes. J Electrochem Soc 1995, 142(9): 2873.

51. Parimalam BS, MacIntosh AD, Kadam R, Lucht BL. Decomposition reactions of anode solid electrolyte interphase (SEI) components with LiPF6. The Journal of Physical Chemistry C 2017, 121(41): 22733-22738. 


\section{Supplementary Files}

This is a list of supplementary files associated with this preprint. Click to download.

- Supplementaryinformation.pdf 